\title{
A Control System for Long Term Investor under Uncertainty based on Fuzzy Logic
}

\author{
Hegazy Zaher \\ Professor of Mathematical Statistics, Institute of \\ Statistical Studies and Research (ISSR), Cairo \\ University, Egypt.
}

\begin{abstract}
This paper introduce a novel control model for long-term investment decision making that faces uncertainty based on fuzzy logic. The proposed control model has the ability to generate accurate recommendations that support long-term investor decisions. This paper presents a creative methodology for dealing with historical data through a deep analysis to explore the strong relation between the age, and the risk of investor. The core idea of this control system is to reduce the number of inputs used in the model. This process is called dimension reduction based on fuzzy techniques. The output of this model is a good allocation for financial resources among three channels: saving, income, and growth.
\end{abstract}

\section{Keywords}

Control system, uncertainty, fuzzy logic, long term investment.

\section{INTRODUCTION}

Many situations of the practical life require the ability to control the risk. This process of control is based on controlling the number of inputs that really effect on the certain output [10].

However there are many variables correlated with other lacked the input analysis process.

The contribution of this paper is introducing a novel control system that achieves minimum risk under certain level of expected return [1]. This paper presents a new method for soft computing risk for investor as decision making support for long -term in money stock [5]. This is considered a significant challenge for investor in financial market. No doubt, the nature of the market has the following characteristics: complicated relationship among variables, dynamic changes, and uncertainty generated from vagueness that effect on the markets of currencies, indexes of financial market and commodities. This is the primary motivator to authors to pay their attention for tackling this issue. This paper distinguish on other previous work based on classical control models .however, the classical control models depend on mathematical basis, the paper faces the significant challenge of uncertainty not directed before to the control systems. The control system hold accurate answers about the following questions: when to saving, rate of income and return growth stocks for minimizing the risk [4].

\author{
Nisren Hassanen Mohamed \\ Ph.D. student, Dept of Operations \\ Researchlnstitute of Statistical Studies and \\ Research (ISSR), Cairo University, Egypt.
}

The deep thought and analysis of the market will help to understand the nature of markets and internal, external factors being able to forecast future movements, and trends. This Control system introduces the real variables that control system after removing the redundant variables which lack the accuracy of prediction.

Author's use this model in the case of determine in the previous searches to compute the minimum risk in portfolio under fixed return [9]

$\operatorname{Min} \sigma_{X}^{2}=\sum_{i=1}^{n} \sum_{j=1}^{n} w_{i} w_{j} \rho_{i j} \sigma_{i} \sigma_{j}$

subject to

$\sum_{i=1}^{n} w_{i} E\left(\mathrm{R}_{I}\right)=E$

$\sum_{i=1}^{n} w_{i}=1.0$

Where:

$\mathrm{R}_{\mathrm{i}=\text { Return on asset } \mathrm{i}}$.

$\mathrm{W}_{\mathrm{i}}=$ Weight of component asset $\mathrm{i}$ (that is, the share of the asset $\mathrm{i}$ in the portfolio).

$\mathrm{W}_{\mathrm{j}}$

$\mathrm{j}=$ Weight of component asset $\mathrm{j}$ (that is, the share of the asset $\mathrm{j}$ in the portfolio).

$\rho_{\mathrm{ij}}=$ correlation coefficient between the rates of return on security $i,{ }_{i}$, and the rates of return on security $j,{ }_{j}$.

$\sigma_{i}=$ standard deviations of ${ }^{r_{i}}$.

$\sigma_{j=\text { standard deviations of }} \mathbf{r}_{j}$

$\mathrm{n}=$ the number of securities. 


\section{MODELING THE CONTROL VARIABLES}

It is usually when deal with Control systems have inputs and outputs the modeler faces the problem of linguistic variables that considered main challenge in this field.

In this section the concept of FLC technique on a system restricted by two inputs C; D and single output is called $\mathrm{M}$. The proposed control system can be extended for Appling it to many practical problems that have more inputs and outputs [6].

The proposed control system is applied within the case of the problem that has many inputs and one output [11], [4].

Linguistic variables are modeled by sets C; D; M; containing certain number of terms $\mathrm{Ci} ; \mathrm{Dj}$; $\mathrm{Mk}$ :

$\mathrm{C}=\{\mathrm{C} 1 \ldots \mathrm{Ci} ; \mathrm{Ci}+1 \ldots \mathrm{Cn}\}$,

$\mathrm{D}=\{\mathrm{D} 1 \ldots \mathrm{Dj} ; \mathrm{Dj}+1 \ldots \mathrm{Dm}\}$

$\mathrm{M}=\{\mathrm{M} 1 \ldots \mathrm{Mk} ; \mathrm{Mk}+1 \ldots \mathrm{Ml}\}$

The terms $\mathrm{Ci}, \mathrm{Dj}$, and $\mathrm{Mk}$ are fuzzy sets defined as

$\mathrm{Ci}=\left\{(\mathrm{x}, \mu \mathrm{Ci}(\mathrm{x})) \mid \mathrm{x} \varepsilon \mathrm{Ci} \_\mathrm{U} 1\right\}, \mathrm{i}=1 \ldots \mathrm{n}$,

$\mathrm{Dj}=\left\{(\mathrm{y}, \mu \mathrm{Dj}(\mathrm{y})) \mid \mathrm{y} \varepsilon \mathrm{Dj} \_\mathrm{U} 2\right\}, \mathrm{j}=1 \ldots \mathrm{m}$,

$\mathrm{Mk}=\left\{(\mathrm{z}, \mu \mathrm{Mk}(\mathrm{z})) \mid \mathrm{z} \varepsilon \mathrm{Mk} \_\mathrm{U} 3\right\}, \mathrm{k}=1 \ldots 1$.

The well -design of the certain sets requires:

(I) Specifying the universal sets U1; U2; U3 of the base variables $\mathrm{x} ; \mathrm{y} ; \mathrm{z}$ for the linguistic variables described by $\mathrm{C}$; $\mathrm{D}$; M.

(ii) Selecting the suitable shape of many shapes, peaks, and different types the membership functions of $\mathrm{Ci} ; \mathrm{Dj}$; Mk. The most common shapes are triangular, trapezoidal, or bellshaped types of membership functions are used.

(iii) Specifying the number of terms, i.e. the numbers $n$; $m$ and 1 .

(iv) Specifying the supporting intervals for the terms $\mathrm{Ci}$; $\mathrm{Dj}$; Mk [3].

\section{RISK CONTROL BY USING FUZZY LOGIC CONTROL (FLC) METHODOLOGY}

The proposed control system deal with the uncertainty generated from vagueness .this model faces the uncertainty by fuzzy logic that is implemented by MATLAB. The first process is defining the input variables which are used in the model. The proposed model can determine the relative importance of these variables .then applying the backward elimination for the lowers. The model generates the rules and returns output variable called Position. The extracted variables are considered the main recommendation for the investor. The model tries to keeps with its simplicity by dimension reduction or reducing variables used in the proposed model. The model keeps only three cases for each variable low, medium and high. The model tries to keeps with its simplicity in the output by the minimum number of rules that describe the model without significant loss for information.

The main merit of this paper is introducing a control system available to investors who have no experiences or knowledge in the field of money stock.

\section{MODELS EXPERIMENTATION}

The Experimentation is based on getting the minimum risk based on the model mean-variance [7], [8]. Then, the checking process for the decision and control system with a fixed return. This work is using the fuzzy logic control methodology for allocating the portions of their investments among three main asset types: savings, income, and growth (asset allocation).

The concepts age and risk tolerance are measured on suitable scales. Age is partitioned into three groups, for instance young ( $\leq 45$ years), middle age (between 45 and 70 years), and old ( $\geq 70$ years). The risk tolerance is partitioned on a psychometric scale from 0 to 100 into low $(\leq 50)$, moderate (between 50 and 80$)$, and high $(\geq 80)$. A questioner filled by the client help financial experts to determine his/her risk tolerance group (low, moderate, or high) [2]. A deficiency in this model is that a person 46 years old is middle age as well as a person who is 60 years old. All ages in the interval [46, 69] have the same status, they equally qualify to be middle age, and there is no gradation level of belonging to the interval. The same is valid for those who are young and old. Similar difficulty arises with the notion of risk tolerance [1].Classical (crisp) models of this type can be improved by using FLC methodology.

The inputs in the fuzzy logic client asset allocation model are age and risk tolerance (risk). The risk can be estimated as in above section and there are three outputs savings, income, and equity. Hence this is a two input and three outputs. The control objective is for any given pair (age, risk) which reflects the state of a client to find how to allocate the asset to savings, income, and growth. Assume that the financial experts describe the two input and three output variables by the terms of triangular and trapezoidal shape as follows [2].

Age $=\{\mathrm{Y}($ young $), \mathrm{MI}($ middle age $), \mathrm{OL}($ old $)\}$.

Risk $=\{\mathrm{L}($ low $), \mathrm{MO}($ moderate $), \mathrm{H}($ high $)\}$.

Saving $=\{\mathrm{L}($ low $), \mathrm{M}($ medium $), \mathrm{H}($ high $)\}$

Income $=\{\mathrm{L}($ low $), \mathrm{M}($ medium $), \mathrm{H}($ high $)\}$.

Growth $=\{\mathrm{L}($ low $), \mathrm{M}($ medium $), \mathrm{H}($ high $)\}$.

They are shown on Figure $1-4$ investment advisory models 


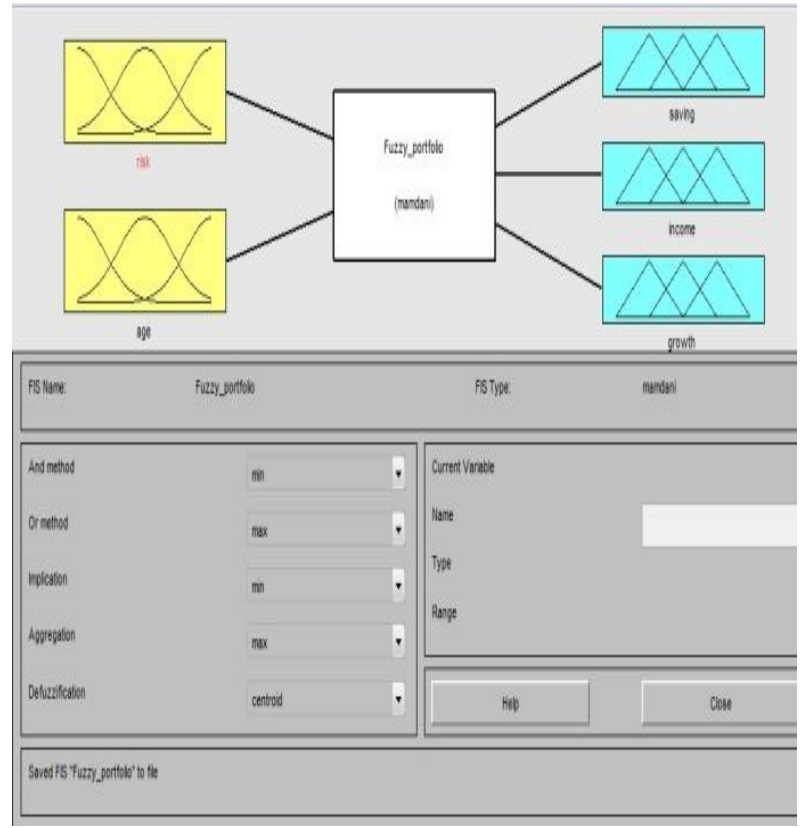

Figure 1.Terms of the input and output.

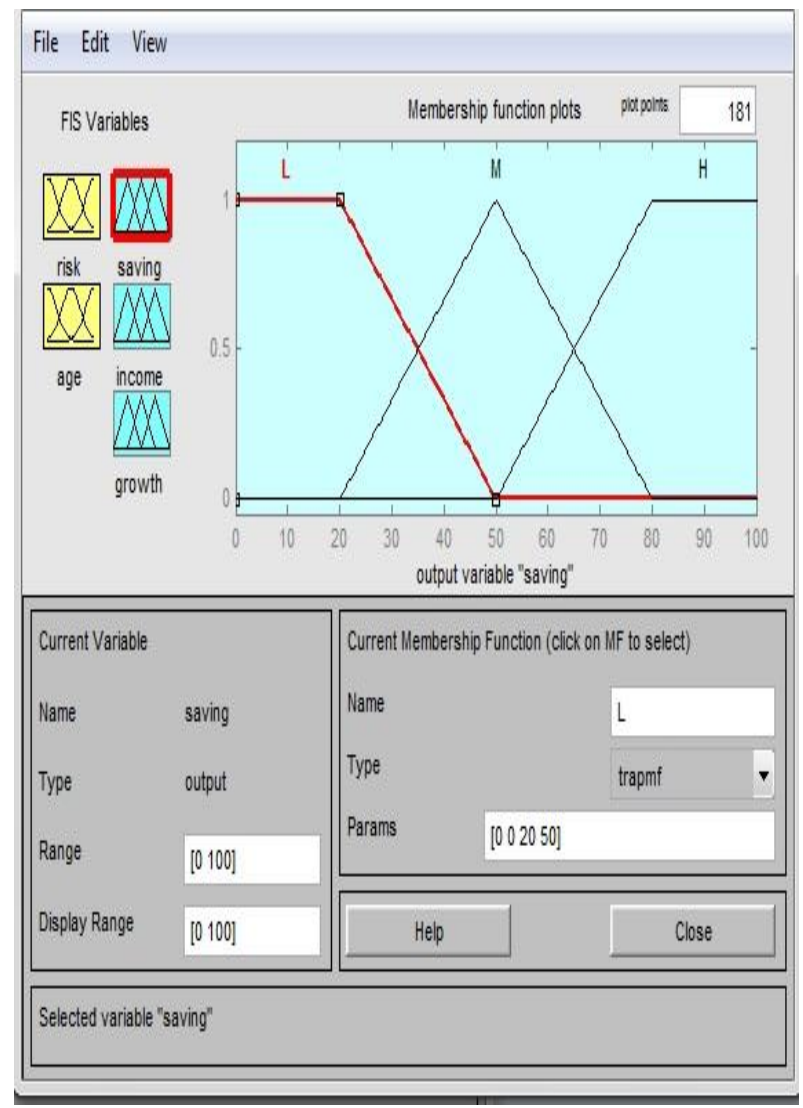

Figure 2.Terms of the input risk tolerance.

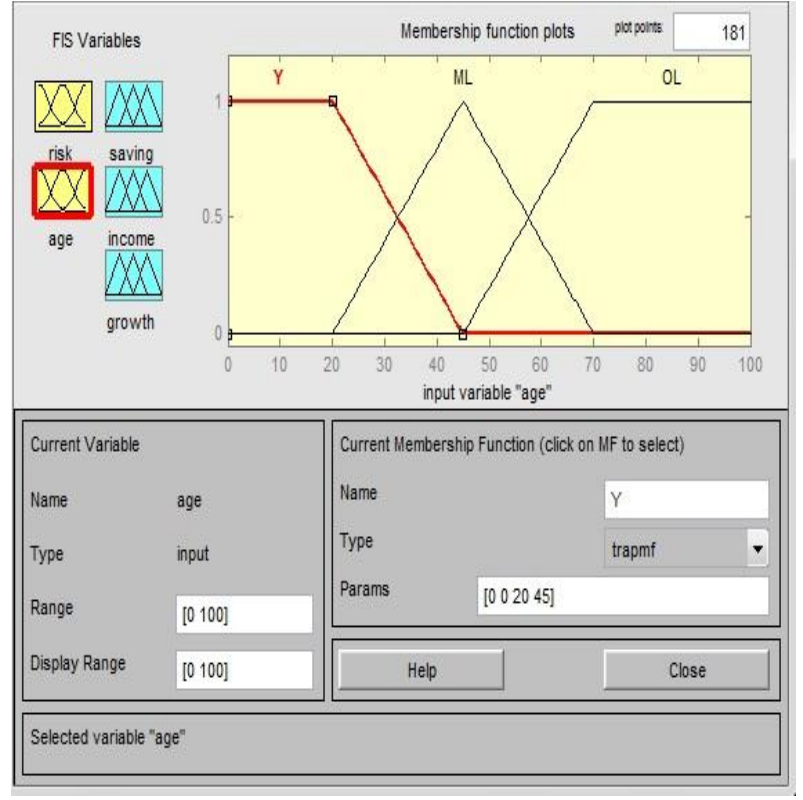

Figure 3.Terms of the input age.

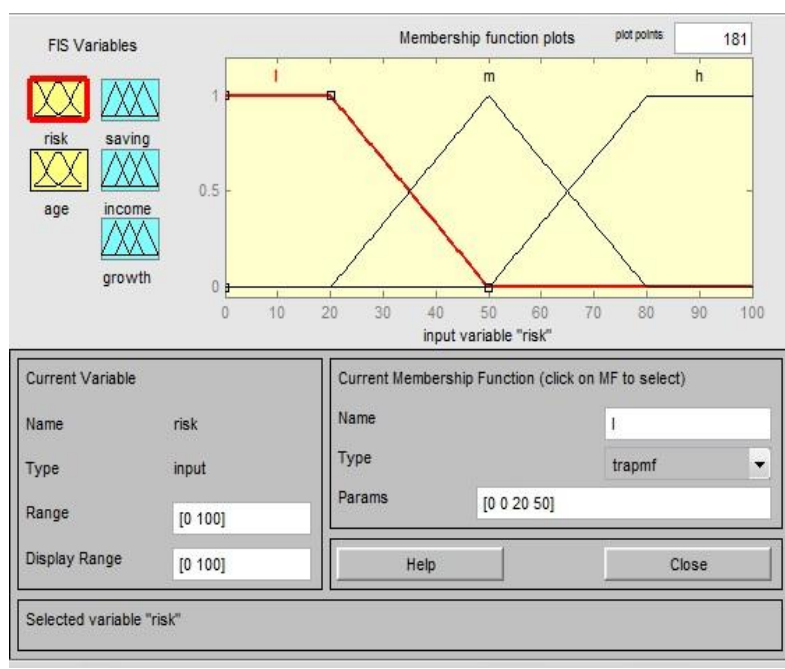

Figure 4. Terms of the output variables savings, income, growth.

The terms of linguistic variables risk, savings, income, and growth are described by the same membership functions as the linguistic variables in the model. The variable age (Figure.3) differs slightly from the other variables; the membership functions of its terms are

$$
\begin{gathered}
\mu \mathrm{Y}(\mathrm{x})=\left\{\begin{array}{cc}
1 & \text { for } \mathrm{x} \leq 20, \\
50-\mathrm{x} & \text { for } 20 \leq \mathrm{x} \leq 50, \\
30 & -
\end{array}\right. \\
\mu \mathrm{MI}(\mathrm{x})=\left\{\begin{array}{cc}
\mathrm{X}-20 & \text { for } 20 \leq \mathrm{x} \leq 50, \\
30 & \\
80-\mathrm{x} & \text { for } 50 \leq \mathrm{x} \leq 80 \\
30 &
\end{array}\right.
\end{gathered}
$$




$$
\mu \mathrm{OL}(\mathrm{x})=\left\{\begin{array}{cl}
\mathrm{X}-50 & \text { for } 50 \leq \mathrm{x} \leq 80 \\
30 & \\
1 & \text { for } 80 \leq \mathrm{x} .
\end{array}\right.
$$

There are nine if ... and ...then rule but each inference rule produces three (not one) conclusions, one for savings, one for income, and one for growth. Consequently the financial experts have to design three decision tables. Assume that these are the tables presented below $1-3$.

\begin{tabular}{l|l|l|l|l|}
\multicolumn{3}{c}{ Risk tolerance } \\
\cline { 3 - 5 } age & & Low & Moderate & High \\
\cline { 2 - 5 } & Young & M & L & L \\
\cline { 2 - 5 } & Middle & M & L & L \\
\hline Old & H & M & M \\
\hline
\end{tabular}

Table 1. Decision table for the output savings.

Risk tolerance

\begin{tabular}{|c|c|c|c|}
\hline & Low & Moderate & High \\
\hline Young & M & M & $\mathrm{L}$ \\
\hline Middle & $\mathrm{H}$ & $\mathrm{H}$ & M \\
\hline Old & $\mathrm{H}$ & $\mathrm{H}$ & $\mathrm{M}$ \\
\hline
\end{tabular}

Table 2 Decision table for the output income.

Risk tolerance

\begin{tabular}{|c|c|c|c|}
\hline & Low & Moderate & High \\
\hline Young & $\mathrm{M}$ & $\mathrm{H}$ & $\mathrm{H}$ \\
\hline Middle & $\mathrm{L}$ & $\mathrm{M}$ & $\mathrm{H}$ \\
\hline Old & $\mathrm{L}$ & $\mathrm{L}$ & $\mathrm{M}$ \\
\hline
\end{tabular}

Table 3. Decision table for the output growth.

They are shown on Figure 5 the strength of the rules calculated using above tables [11].

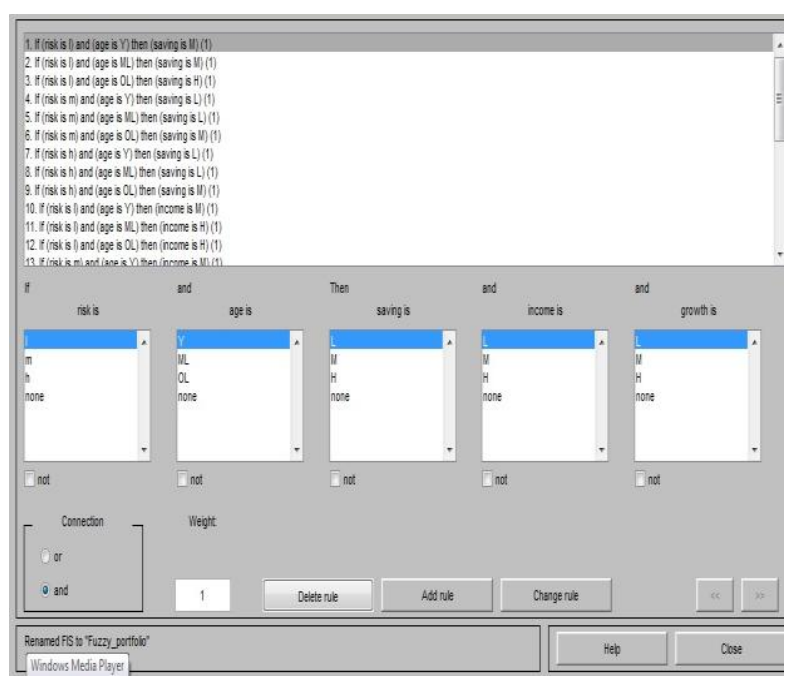

Figure 5 the rules editor.

Show the result after made rules viewers in Figure 6.

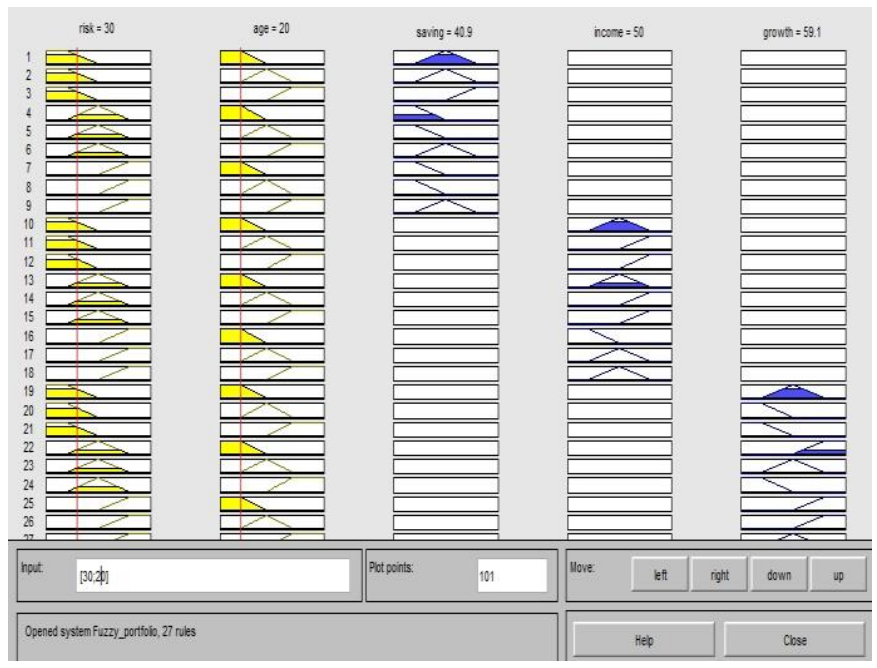

Figure 6 show the result of rules viewers

The following table 4 is the cases for the different client and the decision you should taken

\begin{tabular}{|c|c|c|c|c|}
\hline age & risk & saving & income & growth \\
\hline 20 & 30 & 40.9 & 50 & 59.1 \\
\hline 20 & 50 & 18.3 & 50 & 81.7 \\
\hline 20 & 80 & 18.3 & 18.3 & 81.7 \\
\hline 50 & 20 & 55.4 & 80.6 & 19.4 \\
\hline 50 & 60 & 27.8 & 69.1 & 53.5 \\
\hline 50 & 90 & 26.3 & 50 & 73.7 \\
\hline 75 & 20 & 81.7 & 81.7 & 18.3 \\
\hline 75 & 50 & 50 & 81.7 & 18.3 \\
\hline 75 & 90 & 50 & 50 & 50 \\
\hline
\end{tabular}

Table 4. Risk for different age and risk.

This gives the following asset allocation of the client whose age is 20 and risk tolerance 30 : 
Savings : $40.9 \%$.

Income : $50 \%$.

Growth : $59.1 \%$

Rounding off gives savings $41 \%$, income $50 \%$, and growth $59 \%$.

Applying the above approach and from table 4. It's possible to find linear relation using least square technique between income and other variables. The only significant variable is saving and growth the following is the regression line between income and other variable.

Income $=29.452+0.725 *$ saving, significant 0.03

Income $=94.608-0.701 *$ growth, significant 0.002

Saving $=10.579+0.627 *$ age,$\quad$ significant $0.03 \quad \ldots$ (3)

\section{DISCUSSION AND CONCLUSIONS}

Applying the proposed approach show the following results (table 4) and equations (1), (2) and (3).

1- Saving increases as age increases.

2-There is a significant relation between income and saving. Income increases as saving increases.

3- A highly significant relation between income and growth. Income increases as growth increases.

\section{REFERENES}

[1] Acar, M., D.Karahoca and A. Karahoca (2011)"Designing an Early Warning System for Stock Market Crashes by Using ANFIS".

[2] George, Bojadziev. , Maria, Bojadziev. (2007). " fuzzy logic for business, finance, and management".

[3] George, Bojadziev. , Maria, Bojadziev. and Lotfi, A Zadeh. (1997). "Applications of Fuzzy Logic Control. Fuzzy Logic for Business, Finance, and Management": pp. 157-185.

[4] Chen,p., Pankaj,c. ,Claudia, R (2007), ”Fuzzy MLS: An Experiment on Quantified Risk-Adaptive Access Control".

[5] Doyle J. C., (1989)," Glover K., Khargoneckar P. P., Francis B. A. State space solutions to standard and control problems. IEEE Transactions on Automatic Control, , 34(8):831-847.

[6] Dostál, p., Brož, Z.(2011). "Fuzzy logic investment support on the financial market".

[7] Hegazy, Z., and naglaa, r., and nisren, h. (2014), "Optimizing Local Portfolio Return under Risk In Terms Of International Stock Markets Indices".

[8] Hegazy, Z., nisren, h. (2015),"Minimization of Portfolio Risk using Three Different Methods (A Comparative Study)".

[9] Lotfi, A. Zadeh (1965), "Fuzzy Sets, information and control ", vol.8, pp.338-353.

[10] Ossama Y. Abul-Haggag1, Walied.(2013).” Application of Fuzzy Logic for Risk Assessment using Risk Matrix".

[11] Rajasekaran, s. , vijayalak, S. G.A. (2003)," neural networks, fuzzy logic and genetic algorithm: synthesis and applications " ISBN :978-81-203-2186-1. 